\title{
TESTE DE DETERIORAÇÃO CONTROLADA NA AVALIAÇÃO DO VIGOR DE SEMENTES DE MILHO'
}

\author{
CLAUDEMIR ZUCARELI ${ }^{2}$, CLÁUDIO CAVARIANI ${ }^{3}$, CESAR AUGUSTO GASPARETTO SBRUSSI $^{4}$, JOÃO NAKAGAWA ${ }^{5}$
}

RESUMO - Objetivou-se com este trabalho estudar combinações de tempo e temperatura na adequação do teste de deterioração controlada para avaliação do vigor de sementes de milho com diferentes teores de água. Foram utilizados oito lotes de sementes de milho híbrido CO32, cuja qualidade inicial foi determinada pelos testes de: teor de água, massa de mil sementes, porcentagens de germinação e de plântulas normais na primeira contagem do teste de germinação, massa seca das porções aérea, radicular e total de plântulas, teste de frio, teste de envelhecimento acelerado, teste de condutividade elétrica, tetrazólio, emergência de plântulas no campo e velocidade de emergência de plântulas no campo. A umidade inicial dos lotes de sementes foi ajustada pelo método de imersão em água para 15,20 e $25 \%$. Para cada teor de água foram avaliadas nove combinações de períodos (16, 24 e 48 horas) e temperaturas de deterioração (42, 45 e $\left.48{ }^{\circ} \mathrm{C}\right)$. Após a deterioração determinou-se o teor de água e a porcentagem de germinação das sementes. Os dados foram submetidos à análise de variância e as médias comparadas pelo teste de Tukey e análise de correlação aos $5 \%$ em esquema fatorial $9 \mathrm{X} 8$, sendo nove combinações de tempo e temperatura e oito lotes de sementes. As combinações $24 \mathrm{~h}-45^{\circ} \mathrm{C}, 48 \mathrm{~h}-45^{\circ} \mathrm{C}$ e $16 \mathrm{~h}-45^{\circ} \mathrm{C}$ para sementes com teor de água ajustado para 15,20 e $25 \%$, respectivamente, são eficientes para avaliação do vigor de sementes de milho pelo teste de deterioração controlada.

Termos para indexação: Zea mays, análise de semente, potencial fisiológico, germinação.

\section{CONTROLLED DETERIORATION TEST TO EVALUATE MAIZE SEED VIGOR}

\begin{abstract}
The objective of the present research was to study the combination of time and temperature on the suitability of the controlled deterioration test for evaluating the vigor of maize seed with different water contents. Eight seed lots of hybrid maize CO32 were used, whose initial quality was determined by the following tests: water content, thousand seed weight, electric conductivity, germination, first germination count, seedling total dry matter and of the aerial parts and roots, cold test, accelerated aging, tetrazolium, emergence and rate of emergence in the field. The initial moisture content of seed lots was adjusted by immersion in water to 15,20 and $25 \%$. Nine combinations of periods (16, 24 and 48 hours) and deterioration temperatures $(42,45$ and 48 ${ }^{\circ} \mathrm{C}$ ) were evaluated for each water content. After deterioration, the water content and percentage of germination were determined and the data submitted to ANOVA with means compared by the Tukey test and a correlation analysis to $5 \%$ in a factorial $9 \times 8$, with nine combinations of temperature and time and eight seed lots. The combinations $24-45{ }^{\circ} \mathrm{C}, 48-45^{\circ} \mathrm{C}$ and $16-45{ }^{\circ} \mathrm{C}$ for seeds with water contents adjusted to 15,20 and $25 \%$ respectively, are efficient for evaluating maize seed vigor using a controlled deterioration test.
\end{abstract}

Index terms: Zea mays, seed analysis, potential physiological, germination

${ }^{1}$ Submetido em 04/02/11. Aceito para publicação em 01/04/11. Apresentado ao programa de Pós Graduação em agricultura da FCA/ UNESP

${ }^{2}$ Eng. Agr, Dr. Professor Adjunto, Departamento de Agronomia/ Fitotecnia, Universidade Estadual de Londrina UEL, CxP: 6001, CEP:, 86051-990,Londrina/PR, claudemircca@uel.br

${ }^{3}$ Eng. Agrônomo, Dr. Professor Assistente, Departamento de Produção
Vegetal, UNESP/Botucatu. Cx P.: 237, CEP.: 18610-307, Botucatu/SP. ccaveriani@sca.unesp.br

${ }^{4}$ Eng. Agr. Mestrando, Programa de Pós-graduação em Agronomia, UEL, Bolsista CAPES. cesarsbrussi@yahoo.com.br

${ }^{5}$ Eng. Agrônomo, Dr. Professor Titular aposentado, Departamento de Produção Vegetal, UNESP/Botucatu. Cx P.: 237, CEP.: 18603-970, Botucatu/SP. secdamv@sca.unesp.br 


\section{INTRODUÇÃO}

Os testes de vigor são desenvolvidos com o objetivo de identificar diferenças no potencial fisiológico dos lotes de sementes, principalmente daqueles que apresentam resultados semelhantes e elevados no teste de germinação (Marcos Filho, 1999a). Desta forma, espera-se que o teste de vigor permita distinguir, com segurança, lotes de alto vigor dos de baixo vigor (Marcos Filho, 2005). O nível de vigor das sementes pode afetar o potencial de armazenamento do lote e persistir no campo, influenciando o desenvolvimento da planta, a uniformidade da lavoura e o seu rendimento (Carvalho e Nakagawa, 2000).

A deterioração controlada é um teste de vigor semelhante ao de envelhecimento acelerado, porém com controle mais preciso da temperatura e umidade durante o período de envelhecimento (Hampton e TeKrony, 1995). Desta forma, as sementes são expostas a condições idênticas de deterioração, resultando em maior precisão e eficiência na distinção do potencial fisiológico dos lotes de sementes. O teor de água inicial das sementes de todos os lotes é uniformizado antes da exposição à deterioração (Matthews, 1980) e este, permanece constante durante o período de deterioração, fato não verificado no teste de envelhecimento acelerado (Silveira, 2006).

$\mathrm{O}$ ajuste do teor de água faz com que as sementes atinjam, antecipadamente, o ponto de equilíbrio, sendo submetidas a um estresse mais rigoroso que no teste de envelhecimento, onde o teor de água aumenta descontroladamente entre as mesmas durante o teste, até atingirem o equilíbrio (Krzyzanowski et al., 2001). Considerando que, a deterioração ocorre em função da exposição da semente a temperatura e umidade elevadas por determinado período de tempo, o estudo e identificação da melhor combinação desses fatores é de extrema importância para a padronização do teste (Zucareli, 2002).

Por ser relativamente simples, não exigir investimentos significativos e não apresenta dificuldades acentuadas para padronização (Santos e Menezes, 2003), o teste de deterioração controlada, tem sido utilizado frequentemente para a avaliação do potencial fisiológico de sementes de diversas espécies olerícolas como cenoura (Rodo et al., 2000), tomate (Panobianco e Marcos Filho 2001), brócolis (Mendonça et al., 2003) e beterraba (Silva e Vieira, 2010). Embora em número mais restrito, encontram-se, também, trabalhos referentes à eficiência do teste de deterioração controlada para sementes de grandes culturas como soja
(Rossetto e Marcos Filho, 1995), feijão (Santos et al., 2003), e algodão (Dutra e Medeiros Filho, 2008).

Padilha et al. (2001), utilizando sementes de milho, híbrido duplo AG-122, com três diferentes níveis de teor de água $(15,20$ e $25 \%)$, em períodos de 24 e 48 horas a temperatura constante de $40{ }^{\circ} \mathrm{C}$, concluíram que os teores de água de 15 e $20 \%$, por 24 horas a $40{ }^{\circ} \mathrm{C}$ e de $15 \%$ por 48 horas a $40{ }^{\circ} \mathrm{C}$ mostraram-se adequados para a realização do teste. No entanto, não existem trabalhos com relação à combinação tempo e temperatura para teores específicos de água em sementes de milho.

O teste de deterioração controlada tem apresentado alta relação com a emergência de plântulas no campo para diversas espécies (Matthews e Powell, 1987; Bustamante et al., 1984; Wang e Hampton, 1989). O teste de vigor torna-se útil como ponto de apoio a pesquisadores, indústria e tendem também a subsidiar os produtores. No entanto, a eficiência do teste de deterioração controlada, assim como de qualquer outro, depende de padronização para as diferentes espécies.

Neste contexto, o trabalho teve por objetivo estudar combinações de tempo e temperatura para o teste de deterioração controlada na avaliação do vigor de sementes de milho com diferentes teores iniciais de água.

\section{MATERIAL E MÉTODOS}

O trabalho foi realizado no Laboratório de Análise de Sementes do Departamento de Produção Vegetal da Faculdade de Ciências Agronômicas (FCA), da Universidade Estadual Paulista (UNESP), em BotucatuSP. Foram utilizadas sementes de milho híbrido CO32, peneira 20, não tratadas.

A avaliação da qualidade fisiológica inicial dos lotes de sementes foi realizada pelas seguintes determinações: Teor de água: quatro repetições de 15 sementes inteiras em estufa a $105 \pm 3{ }^{\circ} \mathrm{C}$, durante 24 horas (Brasil, 2009). Massa de mil sementes: determinada com oito repetições de 100 sementes, conforme descrito nas Regras para Análise de Sementes (Brasil, 2009). Germinação e contagem: quatro repetições de 50 sementes, em papel toalha umedecido na proporção de 2,5 vezes a massa do substrato. Os rolos de papel, acondicionados em sacos plásticos, foram mantidos em germinador sob temperatura de $30{ }^{\circ} \mathrm{C}$. A avaliação constou de duas contagens, aos quatro e sete dias após a instalação do teste. A porcentagem de plântulas normais foi computada no quarto dia após a instalação do teste de germinação (Brasil, 2009). Massa seca de plântulas: as 
plântulas normais obtidas na primeira contagem do teste de germinação foram separadas em duas porções, parte aérea e radicular, e secadas a $60 \pm 5^{\circ} \mathrm{C}$ até massa constante. Os resultados foram expressos em grama por plântulas das porções aérea, radicular e total, nesse caso pela somatória das duas anteriores (Nakagawa, 1999). Teste de frio: conduzido utilizando a metodologia do rolo de papel sem solo, com quatro repetições de 50 sementes (Dias e Barros, 1995). Teste de envelhecimento acelerado: utilizaramse quatro repetições de 65 sementes envelhecidas a $42{ }^{\circ} \mathrm{C}$ por 72 horas (Dias e Barros, 1995). Quatro repetições de 15 sementes foram utilizadas para determinação do teor de água e, quatro repetições de 50 sementes foram submetidas ao teste de germinação. Teste de condutividade elétrica: quatro repetições de 25 sementes, previamente pesadas, foram colocadas para embeber em $75 \mathrm{~mL}$ de água destilada, por um período de 24 horas a $25{ }^{\circ} \mathrm{C}$ (Dias e Barros, 1995). Teste de tetrazólio: conduzido empregando-se quatro repetições de 50 sementes conforme metodologia descrita por Dias e Barros (1995). Emergência das plântulas no campo: conduzido com quatro repetições de 50 sementes, semeadas a $5 \mathrm{~cm}$ de profundidade em linhas de $2,5 \mathrm{~m}$, distanciadas de 0,30 m entre si; a avaliação 14 dias após semeadura, considerou as plântulas emergidas, e o resultado expresso em porcentagem (Nakagawa, 1994). Velocidade de emergência das plântulas no campo: conduzido junto com o teste de emergência de plântulas, foram realizadas contagens diárias, a partir da emergência da primeira plântula até o décimo quarto dia. O IVE foi calculado segundo Maguire, citado por (Nakagawa 1999).

Para padronização do teste de deterioração controlada foram empregados oito lotes de sementes de milho, com teor de água inicial ajustado para 15, 20 e $25 \%$. A massa a ser atingida pelas amostras para elevar o teor de água nos níveis desejados, foi previamente determinada tendo como base a massa e os teores de água iniciais das mesmas (Hampton e TeKrony, 1995). O teor de água dos lotes foi ajustado utilizando o método da imersão em água, na qual 100 sementes foram colocadas em recipientes plásticos $(200 \mathrm{~mL})$, imersas em $100 \mathrm{~mL}$ de água destilada com temperaturas de $30{ }^{\circ} \mathrm{C}$, as quais foram mantidas em câmara BOD (Zucareli, 2002).

Para o monitoramento da massa atingida, as sementes foram secadas superficialmente com papel toalha e pesadas periodicamente. Após atingirem a massa pré-determinada para cada teor de água desejado as sementes foram acondicionadas em frascos plásticos que, devidamente vedados, e mantidos à temperatura de $10^{\circ} \mathrm{C}$, por um período de cinco dias, para uniformização do teor de água.

Após a uniformização, as sementes foram acondicionadas em embalagens identificadas de alumínio $(10 \times 15 \mathrm{~cm})$ que, seladas, foram colocadas em banhomaria, nas temperaturas de 42,45 e $48^{\circ} \mathrm{C}$, por períodos de 16, 24 e 48 horas. Essas variáveis foram combinadas em esquema fatorial ( $3 \times 3)$, totalizando nove combinações de tempo e temperatura, com quatro repetições, para cada teor de água considerado.

Decorridos os períodos, as embalagens foram retiradas do banho-maria e mantidas em temperatura ambiente por 30 minutos, para estabilização da temperatura das sementes. Posteriormente foi realizada a determinação do teor de água e da porcentagem de germinação, conforme metodologias descritas.

Os dados foram submetidos à análise de variância e as médias comparadas pelo teste de Tukey aos 5\%, em esquema fatorial 9X8, correspondente a nove combinações tempo/temperatura e oito lotes de sementes, independentemente para cada teor de água. Os resultados do teste de germinação obtidos para cada tratamento após a deterioração controlada, juntamente com os dados da caracterização inicial, foram submetidos a estudo de correlação linear simples a 5\% de significância.

\section{RESULTADOS E DISCUSSÃO}

Os lotes de sementes utilizados no estudo diferiram quanto à qualidade fisiológica inicial, apenas no teor de água, peso de mil sementes, primeira contagem do teste de germinação, teste de frio e massa seca de raiz das plântulas normais obtidas na primeira contagem do teste de germinação (Tabelas 1 e 2). Pela análise conjunta dos testes, apesar da ausência de diferenças significativas entre lotes, a maioria deles, identificou os lotes L1 e L4 como os de menor vigor e L7 e L8 como os de maior vigor. Os demais lotes demonstraram vigor intermediário.

Os lotes apresentaram, inicialmente, alta percentagem de germinação e vigor, condições, segundo Marcos Filho (1999b), adequadas para estudo do teste de deterioração controlada. Segundo o autor, a utilização de lotes de baixa qualidade, com porcentagens de germinação inferior ao valor mínimo exigido para comercialização, contribui para a obtenção de correlações significativas entre os resultados de laboratório e de campo, não detectando eficientemente a influência do vigor sobre o desempenho das sementes. 
TABELA 1. Valores médios do teor de água (TA), peso de mil sementes (PM), porcentagem de germinação (G), primeira contagem do teste de germinação (PC), teste de tetrazólio viabilidade (TZ Via.), teste de frio (TF), envelhecimento acelerado (EA) e teor de água da sementes após o teste de envelhecimento acelerado (TAEA) de oito lotes de sementes de milho híbrido CO32.

\begin{tabular}{ccccccccc}
\hline LOTES & $\begin{array}{c}\text { TA } \\
(\%)\end{array}$ & $\begin{array}{c}\text { PM } \\
(\mathrm{g})\end{array}$ & $\begin{array}{c}\text { G } \\
(\%)\end{array}$ & $\begin{array}{c}\text { TZ via. } \\
(\%)\end{array}$ & $\begin{array}{c}\text { PC } \\
(\%)\end{array}$ & $\begin{array}{c}\text { TF } \\
(\%)\end{array}$ & $\begin{array}{c}\text { EA } \\
(\%)\end{array}$ & $\begin{array}{c}\text { TAEA } \\
(\%)\end{array}$ \\
\hline L1 & $8,1 \mathrm{a}$ & $27,72 \mathrm{ab}$ & $91 \mathrm{a}$ & $96 \mathrm{a}$ & $90 \mathrm{a}$ & $94 \mathrm{a}$ & $35 \mathrm{a}$ & $24,8 \mathrm{a}$ \\
L2 & $8,0 \mathrm{a}$ & $27,78 \mathrm{ab}$ & $90 \mathrm{a}$ & $95 \mathrm{a}$ & $76 \mathrm{~b}$ & $92 \mathrm{a}$ & $45 \mathrm{a}$ & $25,6 \mathrm{a}$ \\
L3 & $7,4 \mathrm{bc}$ & $27,77 \mathrm{ab}$ & $91 \mathrm{a}$ & $98 \mathrm{a}$ & $90 \mathrm{a}$ & $92 \mathrm{a}$ & $39 \mathrm{a}$ & $25,1 \mathrm{a}$ \\
L4 & $7,2 \mathrm{c}$ & $27,55 \mathrm{~b}$ & $94 \mathrm{a}$ & $96 \mathrm{a}$ & $82 \mathrm{ab}$ & $90 \mathrm{ab}$ & $47 \mathrm{a}$ & $24,2 \mathrm{a}$ \\
L5 & $7,7 \mathrm{ab}$ & $28,07 \mathrm{a}$ & $93 \mathrm{a}$ & $97 \mathrm{a}$ & $86 \mathrm{ab}$ & $93 \mathrm{a}$ & $31 \mathrm{a}$ & $24,9 \mathrm{a}$ \\
L6 & $7,9 \mathrm{a}$ & $27,81 \mathrm{ab}$ & $92 \mathrm{a}$ & $98 \mathrm{a}$ & $90 \mathrm{a}$ & $96 \mathrm{a}$ & $38 \mathrm{a}$ & $25,3 \mathrm{a}$ \\
L7 & $7,9 \mathrm{a}$ & $27,53 \mathrm{ab}$ & $94 \mathrm{a}$ & $98 \mathrm{a}$ & $88 \mathrm{a}$ & $96 \mathrm{a}$ & $36 \mathrm{a}$ & $25,7 \mathrm{a}$ \\
L8 & $7,9 \mathrm{a}$ & $27,94 \mathrm{~b}$ & $95 \mathrm{a}$ & $98 \mathrm{a}$ & $88 \mathrm{a}$ & $82 \mathrm{~b}$ & $41 \mathrm{a}$ & $25,1 \mathrm{a}$ \\
\hline CV(\%) & 2.4 & 1.18 & 4.13 & 3.79 & 5.28 & 4.88 & 21.01 & 4.80 \\
\hline
\end{tabular}

Médias seguidas por letras iguais na coluna não diferem pelo teste de Tukey em $5 \%$ de probabilidade.

TABELA 2. Valores médios do teste de condutividade elétrica (CE), teste de tetrazólio vigor (TZVig.), emergência no campo (EC), índice de velocidade de emergência (IVE), massa seca parte aérea (MSPA), massa seca de raiz (MSR) e massa seca total de plântulas do teste de germinação (MST) de oito lotes de sementes de milho híbrido CO32.

\begin{tabular}{lccccccc}
\hline LOTES & $\begin{array}{c}\text { CE } \\
(\mu \mathrm{S} / \mathrm{cm} / \mathrm{g})\end{array}$ & $\begin{array}{c}\text { TZ vig. } \\
(\%)\end{array}$ & $\begin{array}{c}\text { EC } \\
(\%)\end{array}$ & IVE & $\begin{array}{c}\text { MSPA } \\
(\mathrm{g})\end{array}$ & $\begin{array}{c}\text { MSR } \\
(\mathrm{g})\end{array}$ & $\begin{array}{c}\text { MST } \\
(\mathrm{g})\end{array}$ \\
\hline L1 & $21,78 \mathrm{a}$ & $93 \mathrm{a}$ & $86 \mathrm{a}$ & $6,60 \mathrm{a}$ & $0,0042 \mathrm{a}$ & $0,0043 \mathrm{ab}$ & $0,0085 \mathrm{a}$ \\
L2 & $21,34 \mathrm{a}$ & $90 \mathrm{a}$ & $88 \mathrm{a}$ & $7,20 \mathrm{a}$ & $0,0048 \mathrm{a}$ & $0,0048 \mathrm{ab}$ & $0,0096 \mathrm{a}$ \\
L3 & $25,17 \mathrm{a}$ & $97 \mathrm{a}$ & $92 \mathrm{a}$ & $7,11 \mathrm{a}$ & $0,0054 \mathrm{a}$ & $0,0042 \mathrm{~b}$ & $0,0107 \mathrm{a}$ \\
L4 & $24,40 \mathrm{a}$ & $90 \mathrm{a}$ & $90 \mathrm{a}$ & $7,32 \mathrm{a}$ & $0,0045 \mathrm{a}$ & $0,0042 \mathrm{~b}$ & $0,0088 \mathrm{a}$ \\
L5 & $23,10 \mathrm{a}$ & $92 \mathrm{a}$ & $91 \mathrm{a}$ & $7,33 \mathrm{a}$ & $0,0045 \mathrm{a}$ & $0,0043 \mathrm{ab}$ & $0,0089 \mathrm{a}$ \\
L6 & $22,99 \mathrm{a}$ & $92 \mathrm{a}$ & $93 \mathrm{a}$ & $7,54 \mathrm{a}$ & $0,0046 \mathrm{a}$ & $0,0047 \mathrm{ab}$ & $0,0092 \mathrm{a}$ \\
L7 & $21,32 \mathrm{a}$ & $95 \mathrm{a}$ & $95 \mathrm{a}$ & $7,49 \mathrm{a}$ & $0,0045 \mathrm{a}$ & $0,0047 \mathrm{ab}$ & $0,0091 \mathrm{a}$ \\
L8 & $24,08 \mathrm{a}$ & $97 \mathrm{a}$ & $93 \mathrm{a}$ & $7,38 \mathrm{a}$ & $0,0050 \mathrm{a}$ & $0,0049 \mathrm{a}$ & $0,0101 \mathrm{a}$ \\
\hline CV $(\%)$ & 9,10 & 4,30 & 5,14 & 8,65 & 11,19 & 6,64 & 11,19 \\
\hline
\end{tabular}

Médias seguidas por letras iguais na coluna não diferem pelo teste de Tukey em 5\% de probabilidade.

O teor de água dos lotes de sementes para os diferentes teores de água avaliados (15,20 e $25 \%)$ não variou entre as fases do teste de deterioração controlada (umedecimento, uniformização e deterioração), assegurando assim, uniformidade na intensidade do estresse durante a deterioração.

Sementes com teor de água de 15\%: Os maiores valores médios de germinação foram verificados nos tratamentos com menor temperatura, independente do período de deterioração (Tabela 3). Em sementes de soja, também foi verificado que a elevação da temperatura, no teste de envelhecimento acelerado, promove efeitos mais drásticos sobre a germinação que o prolongamento do período de estresse (Bittencourt e Vieira, 2006). O mesmo foi verificado em sementes de arroz (Albuquerque et al., 1995) e milho (Woltz e Tekrony, 2001). José et al. (2004), estudando a tolerância de linhagens de milho à alta temperatura de secagem, verificou que a causa primária 
do dano por altas temperaturas tem sido a desestruturação do sistema de membranas celulares. Larcher (2000) relata que sob baixas temperaturas as membranas ficam mais rígidas e há aumento de energia de ativação necessária para a realização dos processos bioquímicos, interferindo positivamente no processo germinativo das sementes.

TABELA 3. Dados médios de germinação de oito lotes de sementes de milho, híbrido CO32, com teor de água de $15 \%$, após o teste de deterioração controlada com diferentes combinações de tempo e temperatura. Botucatu, 2001.

\begin{tabular}{|c|c|c|c|c|c|c|c|c|c|c|}
\hline \multirow{2}{*}{ Lotes } & \multicolumn{9}{|c|}{ Tempo (hora) / Temperatura $\left({ }^{\circ} \mathrm{C}\right)$} & \multirow{2}{*}{ Médias } \\
\hline & $16-42$ & $16-45$ & $16-48$ & $24-42$ & $24-45$ & $24-48$ & $48-42$ & $48-45$ & $48-48$ & \\
\hline L1 & $89 a b$ & $81 \mathrm{bc}$ & 93 a $\mathrm{AB}$ & 94 a & $73 \mathrm{c} \mathrm{B}$ & 91 a $\mathrm{A}$ & $94 \mathrm{a}$ & $88 \mathrm{ab}$ & $87 \mathrm{abABC}$ & $86 \mathrm{~A}$ \\
\hline L2 & $93 \mathrm{ab}$ & $83 \mathrm{c}$ & $92 \mathrm{abAB}$ & $96 \mathrm{a}$ & 83 c A & $86 \mathrm{bcAB}$ & $94 a b$ & $86 \mathrm{bc}$ & $86 \mathrm{bcABC}$ & $89 \mathrm{~A}$ \\
\hline L3 & $90 \mathrm{ab}$ & $87 \mathrm{ab}$ & 95 a A & $95 \mathrm{a}$ & $85 \mathrm{~b} \mathrm{~A}$ & $86 \mathrm{abAB}$ & $86 a b$ & $88 \mathrm{ab}$ & $93 \mathrm{abA}$ & $90 \mathrm{~A}$ \\
\hline $\mathrm{L} 4$ & $93 \mathrm{ab}$ & $88 \mathrm{abc}$ & $91 \mathrm{abcAB}$ & $94 \mathrm{ab}$ & $86 \mathrm{bc} \mathrm{A}$ & $89 \mathrm{abcAB}$ & 96 a & $88 \mathrm{abc}$ & $83 \mathrm{cBC}$ & $90 \mathrm{~A}$ \\
\hline L5 & $94 \mathrm{a}$ & $85 \mathrm{bc}$ & $89 \mathrm{ab} \mathrm{AB}$ & $93 \mathrm{ab}$ & $89 \mathrm{ab} A$ & $90 \mathrm{ab} \mathrm{AB}$ & $89 a b$ & $93 \mathrm{ab}$ & $79 \mathrm{cC}$ & $89 \mathrm{~A}$ \\
\hline L6 & $90 a b$ & $85 \mathrm{bc}$ & $93 \mathrm{ab} \mathrm{AB}$ & $96 \mathrm{a}$ & 86 bc A & 83 c B & $90 \mathrm{abc}$ & $88 a b c$ & $89 \mathrm{abcAB}$ & $89 \mathrm{~A}$ \\
\hline L7 & $96 a b$ & $85 \mathrm{c}$ & $90 \mathrm{abcAB}$ & $97 \mathrm{a}$ & 90 abc A & $90 \mathrm{abcAB}$ & $91 \mathrm{abc}$ & $88 \mathrm{bc}$ & $84 \mathrm{cABC}$ & $90 \mathrm{~A}$ \\
\hline L8 & $94 \mathrm{ab}$ & $89 \mathrm{abc}$ & 86 bc B & $90 \mathrm{abc}$ & 88 abc A & $90 \mathrm{abcAB}$ & $95 \mathrm{a}$ & $87 \mathrm{abc}$ & $84 \mathrm{c} \mathrm{ABC}$ & $89 \mathrm{~A}$ \\
\hline Médias & $92 a b$ & $85 \mathrm{de}$ & $91 \mathrm{bc}$ & $94 \mathrm{a}$ & $85 \mathrm{e}$ & $88 \mathrm{~cd}$ & $92 \mathrm{ab}$ & $88 \mathrm{~cd}$ & $86 \mathrm{de}$ & \\
\hline
\end{tabular}

Medias seguidas por letras iguais, minúsculas na linha e maiúsculas na coluna, não diferem pelo teste de Tukey em 5\% de probabilidade.

Para Marcos Filho et al. (1987) sementes mais úmidas mostram mais sensibilidade as condições de alta temperatura e umidade relativa, pois apresentam atividade metabólica intensificada, sob as condições de envelhecimento, e as sementes com menor grau de umidade são menos prejudicadas. Desse modo, fixando-se o teor de água é justificado o efeito menos drástico observado nas menores combinações de tempo/temperatura.

Diferenças significativas na germinação entre lotes de sementes de milho foram detectadas, apenas, nos tratamentos $16 \mathrm{~h}-48{ }^{\circ} \mathrm{C}, 24 \mathrm{~h}-45^{\circ} \mathrm{C}, 24 \mathrm{~h}-48{ }^{\circ} \mathrm{C}$ e 48 $\mathrm{h}-48{ }^{\circ} \mathrm{C}$ que, no entanto, não os discriminando de modo semelhante. Contudo, Marcos Filho (1999b) ressalta que um teste pode ser considerado eficiente, mesmo que não seja possível identificar diferenças significativas entre os lotes avaliados, pois estes podem apresentar níveis semelhantes de vigor. O tratamento $16 \mathrm{~h}-48^{\circ} \mathrm{C}$ indicou os lotes L8 e L3 como os de menor e maior vigor, respectivamente. $\mathrm{O}$ tratamento $24 \mathrm{~h}-45{ }^{\circ} \mathrm{C}$ demonstrou como de menor vigor apenas o lote L1; os lotes L7 e L8 apresentaram, juntamente com o lote L5, as maiores porcentagens de germinação. O lote $\mathrm{L} 1$ foi detectado como de maior vigor pelo tratamento $24 \mathrm{~h}-48{ }^{\circ} \mathrm{C}$, que identificou como de menor vigor o lote L6. O tratamento
$48 \mathrm{~h}-48{ }^{\circ} \mathrm{C}$ classificou como de melhor e pior potencial fisiológico os lotes L3 e L5.

Coeficientes de correlação significativos foram observados entre o tratamento $16 \mathrm{~h}-42^{\circ} \mathrm{C}$ com o teste de germinação, $16 \mathrm{~h}-48{ }^{\circ} \mathrm{C}$ e $24 \mathrm{~h}-42{ }^{\circ} \mathrm{C}$ com o teste de frio e $24 \mathrm{~h}-45^{\circ} \mathrm{C}$ com a emergência de plântulas no campo e índice de velocidade de emergência de plântulas (Tabela 4). O tratamento $16 \mathrm{~h}-45^{\circ} \mathrm{C}$ se correlacionou com o teste de condutividade elétrica.

A maioria das combinações avaliadas não mostrou coerência, na classificação dos lotes quanto ao potencial fisiológico, em relação às determinações realizadas inicialmente. Comportamento semelhante, aos inicialmente determinados, foi verificado, apenas, na combinação $24 \mathrm{~h}-45^{\circ} \mathrm{C}$ que, ainda se correlacionou positiva e significativamente com a velocidade e porcentagem de emergência de plântulas no campo. A combinação 24 h-45 ${ }^{\circ} \mathrm{C}$, segundo Powell e Matthews (1981), tem sido uma das mais utilizadas no teste de deterioração controlada.

Sementes com teor de água de $\mathbf{2 0 \%}$ : Todos os tratamentos, com exceção ao $48 \mathrm{~h}-45{ }^{\circ} \mathrm{C}$ e $48 \mathrm{~h}-48{ }^{\circ} \mathrm{C}$, não diferiram entre si em relação a porcentagem de germinação (Tabela 5). $\mathrm{O}$ tratamento $48 \mathrm{~h}-45^{\circ} \mathrm{C}$ foi o único que diferenciou os lotes quanto ao potencial fisiológico, 
indicando os lotes L6 e L7 como os de maior vigor e, os lotes L1 e L2 como os de menor vigor. A combinação do maior período de exposição (48 horas) com as maiores temperaturas $\left(45 \mathrm{e} 48^{\circ} \mathrm{C}\right)$, resultou em porcentagens médias de germinação significativamente menores, em relação aos demais tratamentos. $\mathrm{O}$ tratamento com maior temperatura e tempo de deterioração $\left(48 \mathrm{~h}-48{ }^{\circ} \mathrm{C}\right)$ mostrou-se muito drástico promovendo a morte das sementes. Segundo Aguiar et al. (1993), acima e abaixo dos limites máximos e mínimos de temperatura, respectivamente, pode ocorrer a morte das sementes. Camara et al. (2008) relata que os extremos de temperatura provocam alterações internas nas sementes, dificultando o processo germinativo, sendo tais danos muitas vezes irreversíveis.

TABELA 4. Coeficientes de correlação linear simples entre germinação após deterioração controlada sob diferentes combinações tempo/temperatura e demais testes em sementes de milho com teor de água de $15 \%$.

\begin{tabular}{lccccccccc}
\hline \multirow{2}{*}{ Variáveis } & \multicolumn{7}{c}{ Tempo (hora)/Temperatura $\left({ }^{\circ} \mathrm{C}\right)$} \\
\cline { 2 - 10 } & $16-42$ & $16-45$ & $16-48$ & $24-42$ & $24-45$ & $24-48$ & $48-42$ & $48-45$ & $48-48$ \\
\hline TA & -0.02 & -0.64 & -0.26 & 0.04 & -0.33 & -0.11 & 0.12 & -0.18 & -0.08 \\
PCTG & -0.32 & 0.05 & 0.07 & -0.11 & -0.08 & 0.07 & -0.45 & 0.32 & 0.33 \\
G & $0.72^{*}$ & 0.70 & -0.68 & -0.29 & 0.60 & 0.26 & 0.17 & 0.17 & -0.44 \\
TFRIO & -0.36 & -0.59 & $0.71^{*}$ & $0.87^{*}$ & -0.20 & -0.34 & -0.62 & 0.15 & 0.35 \\
EA & 0.06 & 0.37 & 0.10 & 0.02 & 0.07 & -0.19 & 0.64 & $-0.75^{*}$ & 0.17 \\
TZVIAB. & 0.18 & 0.57 & -0.26 & -0.29 & 0.50 & -0.05 & -0.40 & 0.14 & 0.25 \\
TZVIG. & 0.05 & 0.39 & -0.15 & -0.29 & 0.21 & 0.28 & -0.24 & -0.05 & 0.40 \\
EC & 0.57 & 0.66 & -0.32 & 0.04 & $0.87 *$ & -0.29 & -0.41 & 0.06 & 0.06 \\
IVE & 0.70 & 0.61 & -0.40 & 0.08 & $0.92 *$ & -0.49 & -0.23 & 0.04 & -0.22 \\
CE & -0.13 & $0.76 *$ & 0.07 & -0.46 & 0.31 & 0.05 & -0.11 & 0.13 & 0.22 \\
PSR & 0.36 & 0.06 & -0.48 & -0.11 & 0.27 & -0.40 & 0.27 & -0.51 & -0.02 \\
PSA & -0.04 & 0.43 & 0.16 & -0.17 & 0.34 & -0.09 & -0.30 & -0.24 & 0.55 \\
PST & 0.17 & 0.35 & -0.15 & -0.19 & 0.40 & -0.29 & -0.07 & -0.46 & 0.40 \\
\hline
\end{tabular}

* Significativo aos 5\%. TA: teor de água; PCTG: primeira contagem no teste de germinação; G: Germinação; TFRIO: teste de frio; EA: envelhecimento acelerado; TZVIAB: tetrazólio viabilidade; TZVIG: tetrazólio vigor; EC: emergência a campo; IVE: índice de velocidade de emergência; CE: condutividade elétrica; PSR: peso seco da raiz; PSA: peso seco da parte aérea; PST: peso seco total.

A menor porcentagem de germinação, observada nas combinações dos maiores tempos e temperaturas, baseiase no fato de que o envelhecimento das sementes ocorre mais rapidamente quando apresentam alto teor de água e são armazenadas sob elevada temperatura (Hampton e TeKrony, 1995; Panobianco e Marcos Filho, 1998; Krzyzanowski e Vieira, 1999).

O tratamento $24 \mathrm{~h}-45^{\circ} \mathrm{C}$, combinação mais utilizada segundo Powell e Matthews (1981), resultou em correlação significativa com o teste de frio (Tabela 6). Esta combinação é recomendada para sementes de nabo, de couve-flor, de alface e de ervilha, com teor de água de $20 \%$ (Krzyzanowski e Vieira, 1999). Em sementes de milho, Padma e Reddy
(1998) constataram que, dentre os testes que apresentaram correlação significativa com a emergência, o teste de deterioração controlada, conduzido por 24 horas a 45 ${ }^{\circ} \mathrm{C}$ com teor de água das sementes de $20 \%$, demonstrou melhores resultados, detectando lotes com baixo potencial de emergência de plântulas. Por outro lado, Bhering et al. (2004), constatou que para sementes de melão, o teste de deterioração controlada, conduzido por 24 horas a $45{ }^{\circ} \mathrm{C}$ e com $20 \%$ de umidade não resultou em redução na porcentagem de germinação, quando comparado aos valores obtidos no teste padrão de germinação, não sendo, esta condição, suficiente para provocar a deterioração das sementes. 
TABELA 5. Dados médios de germinação de oito lotes de sementes de milho, híbrido CO32, com teor de água de $\mathbf{2 0} \%$, após o teste de deterioração controlada com diferentes combinações de tempo e temperatura.

\begin{tabular}{|c|c|c|c|c|c|c|c|c|c|c|}
\hline \multirow{2}{*}{ Lotes } & \multicolumn{9}{|c|}{ Tempo (hora) / Temperatura $\left({ }^{\circ} \mathrm{C}\right)$} & \multirow{2}{*}{ Médias } \\
\hline & $16-42$ & $16-45$ & $16-48$ & $24-42$ & $24-45$ & $24-48$ & $48-42$ & $48-45$ & $48-48$ & \\
\hline L1 & $90 \mathrm{a}$ & $86 \mathrm{a}$ & $91 \mathrm{a}$ & $92 \mathrm{a}$ & $92 \mathrm{a}$ & $92 \mathrm{a}$ & $87 \mathrm{a}$ & $41 \mathrm{~b} \mathrm{C}$ & $0 \mathrm{c}$ & $70 \mathrm{~B}$ \\
\hline $\mathrm{L} 2$ & $91 \mathrm{a}$ & $90 \mathrm{a}$ & $91 \mathrm{a}$ & 93 a & $91 \mathrm{a}$ & $91 \mathrm{a}$ & $86 \mathrm{a}$ & 68 b B & $0 \mathrm{c}$ & $71 \mathrm{~A}$ \\
\hline L3 & 93 a & 89 a & 89 a & 93 a & 90 a & 89 a & $86 \mathrm{ab}$ & $76 \mathrm{~b} \mathrm{AB}$ & $1 \mathrm{c}$ & $72 \mathrm{~A}$ \\
\hline L4 & $92 \mathrm{a}$ & $91 \mathrm{a}$ & $87 \mathrm{a}$ & $88 \mathrm{a}$ & $91 \mathrm{a}$ & $87 \mathrm{a}$ & $88 \mathrm{a}$ & $73 \mathrm{~b} \mathrm{AB}$ & $0 \mathrm{c}$ & $71 \mathrm{~A}$ \\
\hline L5 & 93 a & $92 \mathrm{a}$ & 89 a & 93 a & $88 \mathrm{a}$ & 89 a & 89 a & $75 \mathrm{~b} \mathrm{AB}$ & $2 \mathrm{c}$ & $73 \mathrm{~A}$ \\
\hline L6 & $92 \mathrm{a}$ & $88 \mathrm{ab}$ & $86 \mathrm{ab}$ & $91 \mathrm{a}$ & $91 \mathrm{a}$ & $85 \mathrm{ab}$ & $91 \mathrm{a}$ & $79 \mathrm{~b}$ A & $0 \mathrm{c}$ & $72 \mathrm{~A}$ \\
\hline L7 & $93 \mathrm{a}$ & $93 \mathrm{a}$ & $90 \mathrm{a}$ & $90 \mathrm{a}$ & 93 a & $90 \mathrm{a}$ & $87 \mathrm{ab}$ & 79 b A & $0 \mathrm{c}$ & $73 \mathrm{~A}$ \\
\hline L8 & $91 \mathrm{a}$ & $90 \mathrm{a}$ & $90 \mathrm{a}$ & $91 \mathrm{a}$ & $87 \mathrm{a}$ & $90 \mathrm{a}$ & $90 \mathrm{a}$ & $71 \mathrm{~b} \mathrm{AB}$ & $0 \mathrm{c}$ & $71 \mathrm{~A}$ \\
\hline Médias & $92 \mathrm{a}$ & $90 \mathrm{a}$ & 89 a & $92 \mathrm{a}$ & $91 \mathrm{a}$ & 89 a & $88 \mathrm{a}$ & $70 \mathrm{~b}$ & $0 \mathrm{c}$ & \\
\hline \multicolumn{11}{|c|}{$\mathrm{CV}(\%) \quad 3.14$} \\
\hline
\end{tabular}

Medias seguidas por letras iguais, minúsculas na linha e maiúsculas na coluna, não diferem pelo teste de Tukey aos 5\% de probabilidade.

TABELA 6. Coeficientes de correlação linear simples entre germinação após deterioração controlada sob diferentes combinações tempo/temperatura e demais testes em sementes de milho com teor de água de $20 \%$.

\begin{tabular}{lrrrrrrrrr}
\hline \multirow{2}{*}{ Variáveis } & \multicolumn{8}{c}{ Tempo (hora) / Temperatura $\left({ }^{\circ} \mathrm{C}\right)$} \\
\cline { 2 - 10 } & $16-42$ & $16-45$ & $16-48$ & $24-42$ & $24-45$ & $24-48$ & $48-42$ & $48-45$ & $48-48$ \\
\hline TA & -0.08 & -0.25 & 0.50 & 0.20 & 0.14 & 0.50 & 0.16 & -0.38 & -0.13 \\
PCTG & 0.33 & -0.32 & -0.16 & 0.02 & 0.06 & -0.16 & 0.27 & -0.10 & 0.26 \\
G & -0.44 & 0.58 & -0.27 & $-0.81^{*}$ & -0.16 & -0.27 & 0.39 & 0.49 & 0.05 \\
TFRIO & 0.35 & -0.15 & -0.17 & 0.11 & $0.77^{*}$ & -0.17 & -0.33 & -0.02 & -0.07 \\
EA & 0.17 & 0.01 & -0.19 & -0.27 & -0.05 & -0.19 & -0.06 & 0.15 & -0.59 \\
TZVIAB. & 0.25 & 0.16 & -0.23 & -0.14 & -0.33 & -0.23 & 0.40 & 0.42 & 0.33 \\
TZVIG. & 0.40 & 0.02 & 0.23 & -0.06 & -0.24 & 0.23 & -0.02 & 0.09 & 0.08 \\
EC & 0.06 & 0.60 & -0.42 & -0.41 & -0.17 & -0.42 & 0.38 & $0.85^{*}$ & 0.24 \\
IVE & -0.22 & $0.71 *$ & -0.58 & -0.43 & -0.20 & -0.58 & 0.52 & $0.95^{*}$ & 0.25 \\
CE & 0.22 & 0.00 & -0.46 & 0.08 & -0.55 & -0.46 & 0.18 & 0.29 & 0.32 \\
PSR & -0.02 & 0.12 & 0.12 & -0.08 & -0.21 & 0.12 & 0.45 & 0.23 & -0.18 \\
PSA & 0.55 & 0.09 & 0.10 & 0.34 & -0.44 & 0.10 & -0.18 & 0.32 & 0.15 \\
PST & 0.40 & 0.14 & 0.14 & 0.20 & -0.44 & 0.14 & 0.12 & 0.36 & 0.01 \\
\hline
\end{tabular}

*Significativo aos 5\%. TA: teor de água; PCTG: primeira contagem no teste de germinação; G: Germinação; TFRIO: teste de frio; EA: envelhecimento acelerado; TZVIAB: tetrazólio viabilidade; TZVIG: tetrazólio vigor; EC: emergência a campo; IVE: índice de velocidade de emergência; CE: condutividade elétrica; PSR: peso seco da raiz; PSA: peso seco da parte aérea; PST: peso seco total.

Apesar da ampla utilização da combinação 24 h-45 ${ }^{\circ} \mathrm{C}$, a estreita relação existente entre a taxa de deterioração, o teor de água da semente e o período e temperatura de deterioração possibilitam modificações no teste de deterioração controlada (Powell e Matthews, 1981). Desse modo, Dubey et al. (1994) constataram correlação altamente significativa entre o teste de deterioração controlada e emergência de plântulas no campo em sementes de arroz 
com teor de água de $20 \%$ deterioradas a $40{ }^{\circ} \mathrm{C}$ por 48 horas.

O tratamento 48 h- $45{ }^{\circ} \mathrm{C}$ apresentou correlação significativa com a velocidade e o total de emergência de plântulas no campo, revelando-se a combinação mais adequada para a deterioração de sementes com teor de água de 20\% (Tabela 6). Para sementes de berinjela, Demir, et al. (2004), obtiveram resultados compatíveis com os da emergência de plântula em campo após deterioração por 24,48 e 72 horas a $45{ }^{\circ} \mathrm{C}$ e $20 \%$ de água nas sementes. Corroborando com os resultados obtidos Santos et al. (2003), estudando o teste de deterioração controlada para avaliação do vigor de sementes de feijão, constataram que o tratamento com sementes com umidade inicial de
$20 \%$, por 48 horas sob temperatura de $45^{\circ} \mathrm{C}$, permite a separação de sementes de feijoeiro com diferentes níveis de vigor.

Sementes com teor de água de 25\%: As maiores porcentagens médias de germinação, após a deterioração controlada, foram obtidas nas combinações das duas menores temperaturas e tempos de deterioração (Tabela 7). A taxa de deterioração das sementes é aumentada consideravelmente pela exposição a níveis muito adversos de temperatura e umidade relativa do ar (Marcos Filho, 1999b). Considerando que as sementes apresentavam alto teor de água, era esperado que quanto menor a temperatura e tempo de estresse, maior a porcentagem de germinação.

TABELA 7. Dados médios de germinação de oito lotes de sementes de milho, híbrido CO32, com teor de água de $\mathbf{2 5 \%}$, após o teste de deterioração controlada com diferentes combinações de tempo e temperatura.

\begin{tabular}{|c|c|c|c|c|c|c|c|c|c|c|}
\hline \multirow{2}{*}{ Lotes } & \multicolumn{9}{|c|}{ Tempo (hora) / Temperatura $\left({ }^{\circ} \mathrm{C}\right)$} & \multirow{2}{*}{ Médias } \\
\hline & $16-42$ & $16-45$ & $16-48$ & $24-42$ & $24-45$ & $24-48$ & $48-42$ & $48-45$ & $48-48$ & \\
\hline L1 & $89 a$ & $72 \mathrm{ab} B$ & 79 a A & $82 \mathrm{a}$ & $80 \mathrm{a}$ & $53 \mathrm{c} \mathrm{A}$ & $77 \mathrm{a}$ & $57 \mathrm{bc} A B$ & $0 \mathrm{~d}$ & $60 \mathrm{AB}$ \\
\hline L2 & $89 \mathrm{a}$ & $91 \mathrm{a} A B$ & $78 \mathrm{ab} \mathrm{AB}$ & $82 \mathrm{a}$ & $84 \mathrm{a}$ & $41 \mathrm{c} \mathrm{AB}$ & $72 \mathrm{ab}$ & $60 \mathrm{~b} \mathrm{~A}$ & $1 \mathrm{~d}$ & $61 \mathrm{AB}$ \\
\hline L3 & $94 \mathrm{a}$ & 95 a A & $75 \mathrm{ab} A B$ & 93 a & $82 \mathrm{a}$ & $52 \mathrm{c} \mathrm{A}$ & $76 \mathrm{ab}$ & $60 \mathrm{bc} A B$ & $0 \mathrm{~d}$ & $63 \mathrm{~A}$ \\
\hline $\mathrm{L} 4$ & $91 \mathrm{a}$ & 92 a $\mathrm{AB}$ & 75 a $\mathrm{AB}$ & $90 \mathrm{a}$ & $87 \mathrm{a}$ & $41 \mathrm{~b} \mathrm{AB}$ & 81 a & $48 \mathrm{~b} \mathrm{ABC}$ & $2 \mathrm{c}$ & $62 \mathrm{AB}$ \\
\hline L5 & $88 \mathrm{ab}$ & 92 a $\mathrm{AB}$ & $73 a b c \mathrm{AB}$ & $91 \mathrm{a}$ & $82 \mathrm{ab}$ & $56 \mathrm{~cd} \mathrm{~A}$ & $68 \mathrm{bc}$ & $44 \mathrm{~d}$ BC & $0 \mathrm{e}$ & $60 \mathrm{AB}$ \\
\hline L6 & $91 \mathrm{a}$ & $91 \mathrm{a} A B$ & $76 \mathrm{ab} A B$ & $88 \mathrm{a}$ & $81 \mathrm{a}$ & 33 с B & $75 \mathrm{ab}$ & $61 \mathrm{~b} \mathrm{~A}$ & $2 \mathrm{~d}$ & $61 \mathrm{AB}$ \\
\hline L7 & $90 \mathrm{a}$ & 95 a A & $60 \mathrm{~b} \mathrm{~B}$ & 93 a & $83 \mathrm{a}$ & $32 \mathrm{c} \mathrm{B}$ & $82 \mathrm{a}$ & 38 c C & $0 \mathrm{~d}$ & $56 \mathrm{~B}$ \\
\hline L8 & $86 a b$ & 95 a A & $71 \mathrm{~b} \mathrm{AB}$ & $89 a b$ & $84 a b$ & $43 \mathrm{c} \mathrm{AB}$ & $78 \mathrm{ab}$ & $49 \mathrm{c} \mathrm{ABC}$ & $1 \mathrm{~d}$ & $60 \mathrm{AB}$ \\
\hline Médias & $90 \mathrm{a}$ & $90 \mathrm{a}$ & $74 \mathrm{c}$ & $88 \mathrm{a}$ & $83 \mathrm{ab}$ & $44 \mathrm{e}$ & $76 \mathrm{bc}$ & $52 \mathrm{~d}$ & $1 \mathrm{f}$ & \\
\hline
\end{tabular}

Medias seguidas por letras iguais, minúsculas na linha e maiúsculas na coluna, não diferem pelo teste de Tukey aos 5\% de probabilidade.

A diferenciação quanto ao vigor, foi verificada nos tratamentos $16 \mathrm{~h}-48{ }^{\circ} \mathrm{C}, 24 \mathrm{~h}-48{ }^{\circ} \mathrm{C}$ e $48 \mathrm{~h}-45{ }^{\circ} \mathrm{C}$, entretanto, esses não apresentaram comportamento semelhante em relação a classificação inicial dos lotes.. O tratamento $16 \mathrm{~h}-45^{\circ} \mathrm{C}$ detectou como de maior vigor os lotes L7, L8 e L3 e de menor vigor o L1. A combinação $24 \mathrm{~h}-42{ }^{\circ} \mathrm{C}$, apesar da ausência de diferenças estatísticas entre lotes, resultou em maiores e menores porcentagens de germinação para os lotes L7 e L1.

O teor de água de $25 \%$ mostrou-se muito drástico para as combinações $24 \mathrm{~h}-48{ }^{\circ} \mathrm{C}, 48 \mathrm{~h}-45^{\circ} \mathrm{C}$ e principalmente $48 \mathrm{~h}-48{ }^{\circ} \mathrm{C}$, o qual resultou em ausência de germinação, possivelmente em função da drasticidade do tratamento e do alto teor de água da semente durante o processo. De acordo com Krzyzanowski e Vieira (1999), existe um teor de água, tempo e temperatura, que não acarrete morte da totalidade das sementes dos diferentes lotes. Deste modo a combinação $48 \mathrm{~h}-48{ }^{\circ} \mathrm{C}$ com $25 \%$ de umidade mostrou-se inadequada para sementes de milho. Padilha et al. (2001) ressaltaram que quando as sementes de milho foram submetidas com teores mais elevados de água ao teste de deterioração controlada, apresentaram menores porcentagens de germinação, além de sofrerem alterações nos seus padrões enzimáticos, com efeito deletério para as enzimas estudadas.

Os tratamentos 16 h- $42{ }^{\circ} \mathrm{C}, 24 \mathrm{~h}-48{ }^{\circ} \mathrm{C}, 48 \mathrm{~h}-42{ }^{\circ} \mathrm{C}$ 
e 48 h-48 ${ }^{\circ} \mathrm{C}$ não se correlacionaram significativamente com nenhum dos testes iniciais (Tabela 8). Os tratamentos $16 \mathrm{~h}-48{ }^{\circ} \mathrm{C}$ e $48 \mathrm{~h}-45{ }^{\circ} \mathrm{C}$ correlacionaram-se de forma significativa, entretanto negativamente com o teste padrão de germinação. Coeficientes significativos e positivos com a emergência no campo e índice de velocidade de emergência foram observados pelo tratamento 16h$45{ }^{\circ} \mathrm{C}$. Correlação significativa também foi encontrada no tratamento $24 \mathrm{~h}-42{ }^{\circ} \mathrm{C}$, porém com a emergência de plântulas.

TABELA 8. Coeficientes de correlação linear simples entre germinação após deterioração controlada sob diferentes combinações tempo/temperatura e demais testes em sementes de milho com teor de água de $25 \%$.

\begin{tabular}{lrrrrrrrrr}
\hline \multirow{2}{*}{ Variáveis } & \multicolumn{8}{c}{ Tempo (hora) / Temperatura $\left({ }^{\circ} \mathrm{C}\right)$} \\
\cline { 2 - 10 } & $16-42$ & $16-45$ & $16-48$ & $24-42$ & $24-45$ & $24-48$ & $48-42$ & $48-45$ & $48-48$ \\
\hline TA & -0.64 & -0.40 & -0.09 & -0.56 & -0.52 & -0.17 & -0.19 & 0.07 & -0.06 \\
PCTG & 0.22 & -0.13 & -0.17 & 0.39 & -0.69 & 0.11 & 0.19 & -0.03 & -0.03 \\
G & -0.23 & 0.48 & $-0.77^{*}$ & 0.70 & 0.36 & -0.41 & 0.58 & $-0.79 *$ & 0.30 \\
TFRIO & 0.62 & -0.21 & 0.02 & -0.01 & -0.46 & -0.10 & -0.19 & 0.16 & -0.69 \\
EA & 0.14 & 0.25 & 0.22 & -0.20 & $0.84 *$ & -0.39 & 0.43 & 0.28 & 0.51 \\
TZVIAB. & 0.01 & 0.51 & -0.45 & 0.67 & -0.25 & -0.15 & 0.26 & -0.16 & 0.21 \\
TZVIG. & -0.01 & 0.32 & -0.38 & 0.47 & -0.25 & 0.06 & 0.34 & -0.09 & 0.01 \\
EC & 0.02 & $0.85^{*}$ & -0.71 & $0.79 *$ & 0.11 & -0.54 & 0.26 & -0.36 & 0.12 \\
IVE & -0.09 & $0.85^{*}$ & -0.58 & 0.60 & 0.36 & -0.66 & 0.10 & -0.36 & 0.25 \\
MSPL & 0.65 & 0.31 & 0.09 & 0.48 & -0.19 & 0.34 & 0.01 & 0.28 & -0.25 \\
CE & 0.39 & 0.40 & 0.23 & 0.49 & 0.29 & 0.28 & 0.12 & 0.16 & 0.42 \\
PSR & -0.62 & 0.28 & -0.27 & -0.23 & 0.08 & -0.60 & 0.10 & 0.06 & 0.43 \\
PSA & 0.17 & 0.57 & 0.05 & 0.27 & 0.09 & 0.13 & -0.06 & 0.36 & 0.04 \\
PST & -0.23 & 0.57 & -0.11 & 0.07 & 0.11 & -0.23 & 0.01 & 0.30 & 0.27 \\
\hline
\end{tabular}

* Significativo aos 5\%. TA: teor de água; PCTG: primeira contagem no teste de germinação; G: Germinação; TFRIO: teste de frio; EA: envelhecimento acelerado; TZVIAB: tetrazólio viabilidade; TZVIG: tetrazólio vigor; EC: emergência a campo; IVE: índice de velocidade de emergência; CE: condutividade elétrica; PSR: peso seco da raiz; PSA: peso seco da parte aérea; PST: peso seco total.

A combinação 24 h- $45{ }^{\circ} \mathrm{C}$ correlacionou-se com o envelhecimento acelerado, mas não classificou os lotes quanto ao potencial fisiológico. Por outro lado, Torres (2002) e Panobianco e Marcos Filho (2001), que, estudando diferentes condições de deterioração controlada em sementes de melão e tomate, respectivamente, verificaram que o teor de água de $24 \%$ e o período de $24 \mathrm{~h}$ à temperatura de $45{ }^{\circ} \mathrm{C}$ mostraram-se sensibilidade suficiente para a avaliação do potencial fisiológico das sementes.

Assim, as combinações $16 \mathrm{~h}-45^{\circ} \mathrm{C}$ e $24 \mathrm{~h}-42{ }^{\circ} \mathrm{C}$ mostraram, em relação à classificação inicial, coerência na classificação de lotes de sementes de milho deterioradas com teor de água de $25 \%$, detectando lotes de alto e baixo potencial fisiológico e apresentaram correlação significativa com a emergência de plântulas no campo. Contudo, o tratamento $16 \mathrm{~h}-45{ }^{\circ} \mathrm{C}$ revelou-se como o mais adequado, pois apresentou diferenças estatísticas entre lotes, correlacionou-se, também, com a velocidade de emergência de plântulas e, ainda, apresentou maior coeficiente de correlação com porcentagem de emergência de plântulas no campo.

\section{CONCLUSÃO}

As combinações $24 \mathrm{~h}-45{ }^{\circ} \mathrm{C}, 48 \mathrm{~h}-45^{\circ} \mathrm{C}$ e $16 \mathrm{~h}-45$ ${ }^{\circ} \mathrm{C}$ para sementes com teor de água ajustado para 15, 20 e $25 \%$, respectivamente, mostraram-se adequadas para avaliação do vigor de sementes de milho pelo teste de deterioração controlada, correlacionando-se positiva 
e significativamente com a velocidade e o total de emergência de plântulas no campo.

\section{REFERÊNCIAS}

AGUIAR,I.B.;PIÑA-RODRIGUES,F.C.M.;FIGLIOLIA, M.B. Sementes florestais tropicais, ABRATES, Brasília: 350p. 1993.

ALBUQUERQUE, M.C.F.; CAMPOS, C.V.; MENDONÇA, E.A.F.; CALDEIRA, S.A.F.; BRUNCA, R.H.C.G. Teste de envelhecimento acelerado em sementes de arroz: influência da temperatura e do período de exposição. Agricultura Tropical, v.1, n.1, p.9-16, 1995.

BHERING，M.C.; DIAS，D.C.F.S.; TOKUHISA，D.; DIAS, L.A.S. Avaliação do vigor de sementes de melão pelo teste de deterioração controlada. Revista Brasileira de Sementes, v.26, n.1, p.125-129, 2004. http://www. agricultura.gov.br/images/MAPA/arquivos_portal/ACS/ sementes web.pdf

BITTENCOURT, S.R.M.; VIEIRA, R.D. Temperatura e período de exposição de sementes de milho no teste de envelhecimento acelerado. Revista Brasileira de Sementes, v.28, n.3, p.61-168, 2006. http://www.scielo. br/pdf/rbs/v28n3/23.pdf

BRASIL. Ministério da Agricultura, Pecuária e Abastecimento. Regras para análise de sementes. Ministério da Agricultura, Pecuária e Abastecimento. Secretaria de Defesa Agropecuária. Brasília, DF: MAPA/ ACS, 2009. 395p.

BUSTAMANTE, L. et al. Pea seed quality and seedling emergence in the field. Seed Science and Technology,v.12, n.2, p.551-558, 1984.

CARVALHO, N.M.; NAKAGAWA, J. Sementes: ciência, tecnologia e produção. Jaboticabal: FUNEP, 2000. 429p.

CAMARA, C.A.; ARAÚJO NETO, J.C. FERREIRA, V.M.; ALVES, E.U.; MOURA, F.B.P Caracterização morfométrica de frutos e sementes e efeito da temperatura na germinação de parkia pendula (willd) benth. Ex walp. Ciência Florestal, v.18, n.3, p.281-291, 2008. http:// redalyc.uaemex.mx/pdf/534/53418301.pdf

DEMIR, I.; OZDEN, Y.S.; YILMAZ, K. Accelerated ageing test of aubergine, cucumber and melon seeds in relation to time and temperature variables. Seed Science and Technology, v.32, p.851-855, 2004.

DIAS, M.C.L.L.; BARROS, A.S.R. Avaliação da qualidade de sementes de milho. Londrina: IAPAR,
1995. 43p.

DUBEY, A.K.; SINGH, P.; KATIYAR, R.P. Seed vigour in lentil (Lens culinaris Medikus). Seed Research, v.22, n.1, p.74-6, 1994.

DUTRA, A.S.; MEDEIROS FILHO, S. Teste de deterioração controlada na determinação do vigor em sementes de algodão. Revista Brasileira de Sementes, v.30, n.1, 2008. http://www.scielo.br/scielo. php?script $=$ sci $\operatorname{arttext\& pid=S0101-31222008000100003}$

HAMPTON, J.G.; TEKRONY, D.M. Controlled deterioration test. In: HAMPTON, J.G., TEKRONY, D.M. Handbook of vigour test methods. Zurich: International Seed Testing Association, 1995. p.70-78.

JOSÉ, S.C.B.R.; PINHO, E.V.R.V.; PINHO, G.V.; SILVEIRA, C.M. Tolerância de sementes de linhagens de milho à alta temperatura de secagem. Ciência e Agrotecnologia, v.28, n.5, p.1107-1114, 2004. http:// www.editora.ufla.br/site/ adm/upload/revista/28-52004_19.pdf

KRZYZANOWSKI, F.C.; WEST, S.H.; FRANÇA NETO, J.B. O teste de deterioração controlada para avaliação da qualidade fisiológica de sementes de soja. Informativo Abrates, v.11, n.2, p.185, 2001.

KRZYZANOWSKI, F.C.; VIEIRA, R.D. Deterioração controlada. In: KRZYZANOWSKI, F.C.; VIEIRA, R.D.; FRANÇA NETO, J.B. Vigor de sementes: conceitos e testes. Londrina: ABRATES, 1999. p.6.1-6.8.

LARCHER, W. Ecofisiologia vegetal. São Carlos: Rima, 2000. 531p.

MARCOS FILHO, J. Fisiologia de sementes de plantas cultivadas. Piracicaba: FEALQ, 2005. 495p.

MARCOS FILHO, J. Teste de envelhecimento acelerado. In: KRZYZANOWSKI, F.C.; VIEIRA, R.D. FRANÇA NETO, J.B. Vigor de sementes: conceitos e testes. Londrina, ABRATES, 1999b. p.3.1-3.24.

MARCOS FILHO, J. Testes de vigor: importância e utilização. In: KRZYZANOWSKI, F.C.;VIEIRA, R.D.; FRANÇA NETO, J.B. Vigor de sementes: conceitos e testes. Londrina: ABRATES, 1999a. p.1.1-1.21

MARCOS-FILHO, J.; CICERO, S.M.; SILVA, W.R. Avaliação da qualidade fisiológica das sementes. Piracicaba: FEALQ, 1987. 230p.

MATTHEWS, S.; POWEL, A.A. Controlled deterioration test. In PERRY, D. A. (Ed.). Handbook of vigour test methods. Zurich: ISTA, 1987. p.49-56. 
MATTHEWS, S. Controlled Deterioration: A new vigour test for croop seeds. In: HEBBLETHWAITE, P.D. Seed production. London: Butterworths and Co Ltd, 1980. p.647-660.

MENDONÇA, E.A.F.; RAMOS, N.P.; FESSEL, S.A. Adequação da metodologia do teste de deterioração controlada em sementes de brócolis (Brassica oleracea L. - var. italica). Revista Brasileira de Sementes, v.25, n.1, p.18-24, 2003. http://www.scielo.br/scielo. php?pid=S0101-31222003000100004\&script $=$ sci_arttext

NAKAGAWA, J. Testes de vigor baseados na avaliação das plântulas. In: VIEIRA, R.D.; CARVALHO, N.M. (Ed.). Testes de vigor de sementes. Jaboticabal: FUNEP, 1994. 164p.

NAKAGAWA, J. Testes de vigor baseados no desempenho de plântulas. In: KRZYZANOWSKI, F.C.; VIEIRA, R.D.; FRANÇA NETO, J.B. Vigor de sementes: conceitos e testes. Londrina: ABRATES, 1999. p.2.1-2.24.

PADILHA, L.; VIEIRA, M.G.G.C.; VON PINHO, E.V.R. Relação entre o teste de deterioração controlada e o desempenho de sementes de milho em diferentes condições de estresse. Revista Brasileira de Sementes, v.23, n.1, p.198-204, 2001. http://www.abrates.org.br/ revista/artigos/2001/v23n1/artigo28.pdf

PADMA, V.,: REDDY, B.M. Standartization of laboratory vigour test for maize. Seed Research, v.26, p.134-7, 1998.

PANOBIANCO, M.; MARCOS FILHO, J. Comparação entre métodos para avaliação da qualidade fisiológica de sementes de pimentão. Revista Brasileira de Sementes, v.20, p.306-10, 1998. http://www.abrates.org.br/revista/ artigos/1998/v20n2/artigo11.pdf

PANOBIANCO, M.; MARCOS FILHO, J. Envelhecimento acelerado e deterioração controlada em sementes de tomate. Scientia Agrícola, v.58, n.3, p.525531, 2001. http://www.scielo.br/scielo.php?script=sci arttext\&pid $=$ S0103-90162001000300014

POWELL. A.A.; MATTHEWS, S. Evaluation of controlled deterioration: a new vigour test for small seed vegetable. Seed Science Technology, v.9, n.22, p.63340, 1981.

RODO, A.B.; PANOBIANCO, M.; MARCOS FILHO, J. Metodologia alternativa do teste de envelhecimento acelerado para sementes de cenoura. Scientia Agricola, v.57, n.2, p.289-292, 2000. http://www.scielo.br/pdf/sa/ v57n2/v57n2a15.pdf
ROSSETTO, C.A.V.; MARCOS FILHO, J. Comparação entre os métodos de envelhecimento acelerado e deterioração controlada para avaliação da qualidade fisiológica de sementes de soja. Scientia Agricola, v.52, n.1, p.99-105, 1995. http://www.scielo.br/pdf/sa/ v52n1/19.pdf

SANTOS, C.M.R.; MENEZES, N.L.; VILLELA, F.A. Teste de deterioração controlada para avaliação do vigor de sementes de feijão. Revista Brasileira de Sementes, v.25, n.2, p.28-35, 2003. http://www.scielo.br/scielo. php? script $=$ sci arttext $\&$ pid $=S 0101-31222003000400005$

SILVA, J.B.; VIEIRA, R.D. Deterioração controlada em sementes de beterraba. Revista Brasileira de Sementes, v.32, n.1, p.69-76, 2010. http://www.scielo.br/pdf/rbs/ v32n1/v32n1a08.pdf

SILVEIRA, C.M. Teste de deterioração controlada em sementes de amendoim. 2006. 26f. Dissertação (Mestrado em Agronomia) - Universidade Estadual Paulista "Julio de Mesquita Filho" Faculdade de Ciências Agronômicas, Jaboticabal, 2006. http://www.fcav.unesp.br/download/ pgtrabs/pts/m/2809.pdf

TORRES, S.B. Métodos para avaliação do potencial fisiológico de sementes de melão. 2002. 103f. Tese (Doutorado em Agronomia), Escola Superior de Agricultura "Luiz de Queiroz", Universidade de São Paulo, Piracicaba, 2002. http://www.teses.usp.br/teses/ disponiveis/11/11136/tde-23102002-151357/pt-br.php

TORRES, S.B.; CASEIRO, R.F.; RODO, A.B.; MARCOS FILHO, J. Testes de vigor em sementes de maxixe (Cucumis anguria L.) com ênfase ao teste de condutividade elétrica. Revista Brasileira de Sementes, v.20, n.2, p.241-244, 1998. http://www.abrates.org.br/ revista/artigos/1998/v20n2/artigo39.pdf

ZUCARELI, C. Teste de deterioração controlada para avaliação do vigor de sementes de milho (Zea mays L.). 2002. 111f. Dissertação (Mestrado em Agronomia)Universidade Estadual Paulista "Julio de Mesquita Filho" Faculdade de Ciências Agronômicas, Botucatu, 2002.

WANG, Y.R., HAMPTON, J.G. Red clover (Trifolium pratense L.) seed quality. Proceedings Agronomy Society New Zealand, v.19, p.63-8, 1989.

WOLTZ, J.M.; TEKRONY, D.M. Accelerated aging test for corn seed. Seed Technology, v.23, n.1, p.21-34, 2001. 